\title{
Nonvanishing Preservers and Compact Weighted Composition Operators between Spaces of Lipschitz Functions
}

\author{
Dongyang Chen, ${ }^{1}$ Lei Li, $^{2}$ Risheng Wang, ${ }^{2}$ and Ya-Shu Wang ${ }^{3}$ \\ ${ }^{1}$ School of Mathematical Sciences, Xiamen University, Xiamen 361005, China \\ ${ }^{2}$ School of Mathematical Sciences and LPMC, Nankai University, Tianjin 300071, China \\ ${ }^{3}$ Department of Applied Mathematics, Chung Yuan Christian University, Chung-Li 32023, Taiwan
}

Correspondence should be addressed to Lei Li; leilee@nankai.edu.cn

Received 14 June 2013; Accepted 9 September 2013

Academic Editor: Fabio M. Camilli

Copyright (C) 2013 Dongyang Chen et al. This is an open access article distributed under the Creative Commons Attribution License, which permits unrestricted use, distribution, and reproduction in any medium, provided the original work is properly cited.

\begin{abstract}
We will give the $\alpha$-Lipschitz version of the Banach-Stone type theorems for lattice-valued $\alpha$-Lipschitz functions on some metric spaces. In particular, when $X$ and $Y$ are bounded metric spaces, if $T: \operatorname{Lip}(X) \rightarrow \operatorname{Lip}(Y)$ is a nonvanishing preserver, then $T$ is a weighted composition operator $T f=h \cdot f \circ \varphi$, where $\varphi: Y \rightarrow X$ is a Lipschitz homeomorphism. We also characterize the compact weighted composition operators between spaces of Lipschitz functions.
\end{abstract}

\section{Introduction}

The classical Banach-Stone theorem tells us that, when $X$ and $Y$ are compact Hausdorff spaces, every linear surjective isometry from $C(X)$ onto $C(Y)$ can be written as a weighted composition operator; that is, it is of the form

$$
(T f)(y)=J(y) f(\varphi(y)),
$$

where $\varphi$ is a homeomorphism from $Y$ onto $X$ and $J \in$ $C(Y)$ with $|J(y)|=1$ for all $y \in Y$. The theorem has many variable extensions concerning isometries, algebra isomorphisms, and disjointness preserving mappings between continuous function spaces; and we refer the surveys $[1,2]$ for more history about Banach-Stone theorems. Moreover, Kamowitz [3] gave the representation and spectrum of the compact weighted composition operators on the continuous functions.

Cao et al. stated a lattice version of the classical BanachStone theorem in [4]. Later, Chen et al. [5], Ercan and Önal $[6,7]$, and Miao et al. [8] generalized this result. When $X, Y$ are compact Hausdorff spaces and $E, F$ are Banach lattices, by the main results of $[5,7]$, we can see that every vector lattice isomorphism $T$ from $C(X, E)$ onto $C(Y, F)$ preserving the nonvanishing functions must be a weighted composition operator.
Garrido and Jaramillo $[9,10]$ and Weaver [11] tackled the Banach-Stone type theorem for lattices of real Lipschitz functions. Later, Jiménez-Vargas and Villegas-Vallecillos [12] proved that two little Lipschitz algebras are order isomorphic if and only if the corresponding compact metric spaces are Lipschitz homeomorphic. Recently, Jiménez-Vargas et al. [13] presented a Lipschitz version of the result in [5], in which the underlying spaces should be compact.

Our first goal of this paper is to prove the Banach-Stone type theorem in the setting of lattice-valued $\alpha$-Lipschitz functions. Section 2 is devoted to the preliminaries about vector lattices and $\alpha$-Lipschitz functions. Then we will give the $\alpha$ Lipschitz version of Banach-Stone theorem in Section 3. In particular, when $X, Y$ are bounded metric spaces, if $T$ : $\operatorname{Lip}(X) \rightarrow \operatorname{Lip}(Y)$ is a nonvanishing preserver, then we will show that $T$ is a weighted composition operator $T f=$ $h \cdot f \circ \varphi$, where $\varphi: Y \rightarrow X$ is a Lipschitz homeomorphism. Our second aim is to give the characterization of compact weighted composition operators on the $\alpha$-Lipschitz functions.

\section{Preliminaries}

An ordered vector space $E$ is said to be a vector lattice if $\max \{x, y\}$ exists for any $x, y$ in $E$. A vector lattice $E$ is said 
to be a Banach lattice if it is complete under its norm $\|\cdot\|$ and satisfies the Riesz law:

$$
|x| \leq|y| \Longrightarrow\|x\| \leq\|y\|,
$$

where $|x|=\max \{x,-x\}$.

Let $(X, d)$ be a metric space and $E$ a Banach space; if $0<$ $\alpha \leq 1$, a function $f$ from $X$ to $E$ is said to be $\alpha$-Lipschitz if

$$
L_{\alpha}(f)=\sup \left\{\frac{\|f(x)-f(y)\|}{d^{\alpha}(x, y)}: x, y \in X, x \neq y\right\}<\infty .
$$

The $\alpha$-Lipschitz function space $\operatorname{Lip}_{\alpha}(X, E)$ is the space of all $E$ valued $\alpha$-Lipschitz functions on $X . \operatorname{Lip}_{\alpha}^{b}(X, E)$ is the Banach space of all bounded $\alpha$-Lipschitz functions $f: X \rightarrow E$ with the $\alpha$-Lipschitz norm

$$
\|f\|=\max \left\{L_{\alpha}(f),\|f\|_{\infty}\right\},
$$

where $\|f\|_{\infty}=\sup \{\|f(x)\|: x \in X\}$. Furthermore, the little Lipschitz space $\operatorname{lip}_{\alpha}(X, E)$ is then defined to be the closed subspace of $\operatorname{Lip}_{\alpha}(X, E)$ of these functions $f$ with the following property: for every $\varepsilon>0$, there exists $\delta>0$ such that $\| f\left(x_{1}\right)-$ $f\left(x_{2}\right) \|<\varepsilon d^{\alpha}\left(x_{1}, x_{2}\right)$ whenever $d\left(x_{1}, x_{2}\right) \leq \delta . \operatorname{lip}_{\alpha}^{b}(X, E)$ is the subspace of $\operatorname{lip}_{\alpha}(X, E)$ consisting of all bounded functions. Notice that when $\alpha=1, \operatorname{Lip}_{\alpha}(X, E)$ is just Lipschitz space $\operatorname{Lip}(X, E)$. Moreover, if $E$ is a Banach lattice, then $\operatorname{Lip}_{\alpha}(X, E)$ is a vector lattice with the usual pointwise order

$$
f \leq g \Longleftrightarrow f(x) \leq g(x), \quad \forall x \in X .
$$

However, $\operatorname{Lip}_{\alpha}^{b}(X, E)$ is not a Banach lattice since $\|\cdot\|$ does not satisfy the Riesz law in general.

A mapping $\varphi$ from $Y$ to $X$ is said to be a $\alpha$-Lipschitz homeomorphism if it is bijective and $\varphi$ and $\varphi^{-1}$ are both $\alpha$ Lipschitz. If $f$ is in $\operatorname{Lip}_{\alpha}(X)$ and $e$ is a vector in $E$, denote by $f \otimes e$ the function $x \mapsto f(x) e$ in $\operatorname{Lip}_{\alpha}(X, E)$. In particular, $1 \otimes e$ denotes the constant function $x \mapsto e$ on $X$. For any function $f$ in $\operatorname{Lip}_{\alpha}(X, E)$, the zero set $\{x \in X: f(x)=0\}$ of $f$ is denoted by $z(f)$ and its cozero set $\{x \in X: f(x) \neq 0\}$ is $\operatorname{coz}(f)$, and $f$ is said to be nonvanishing if $z(f)=\emptyset$. An operator $T: \operatorname{Lip}_{\alpha}(X, E) \rightarrow \operatorname{Lip}_{\alpha}(Y, F)$ is said to be a nonvanishing preserver if

$$
z(f)=\emptyset \Longleftrightarrow z(T f)=\emptyset, \quad \forall f \in \operatorname{Lip}_{\alpha}(X, E) .
$$

$T$ is said to be a Riesz isomorphism if $T(f \vee g)=T f \vee T g$ and $T(f \wedge g)=T f \wedge T g$ for any $f, g \in \operatorname{Lip}_{\alpha}(X, E)$.

\section{Nonvanishing Preservers on Lipschitz Functions}

In this section, our results will be valid (with the same proof) for different kinds of spaces. For this reason we first consider several situations to work in. Throughout this section we will assume that $0<\alpha \leq 1, X, Y$ are metric spaces and $E, F$ are Banach lattices.

Context 1. $A(X, E)=\operatorname{Lip}_{\alpha}^{b}(X, E), A(Y, F)=\operatorname{Lip}_{\alpha}^{b}(Y, F)$.
Context $2(0<\alpha<1)$. $A(X, E)=\operatorname{lip}_{\alpha}^{b}(X, E), A(Y, F)=$ $\operatorname{lip}_{\alpha}^{b}(Y, F)$.

This means that when we refer to $X, Y, A(X, E), A(Y, F)$, we assume that all of them are included at the same time in one of the above two contexts.

Suppose that $X$ is a metric space and $0<\alpha \leq 1$, for any $x_{1} \neq x_{2} \in X$, the function

$$
f(x)=\max \left\{0,1-\frac{d^{\alpha}\left(x, x_{2}\right)}{d^{\alpha}\left(x_{1}, x_{2}\right)}\right\}
$$

belongs to $\operatorname{Lip}_{\alpha}^{b}(X)$. Moreover, if $0<\alpha<1$, then we can find $\beta>0$ such that $\alpha<\beta<1$, and the function

$$
f(x)=\max \left\{0,1-\frac{d^{\beta}\left(x, x_{2}\right)}{d^{\beta}\left(x_{1}, x_{2}\right)}\right\}
$$

belongs to $\operatorname{lip}_{\alpha}^{b}(X)$. The function $f$ defined in (7) or (8) has the property: $0 \leq f \leq 1, f\left(x_{1}\right)=0, f\left(x_{2}\right)=1$ and $z(f)=$ $\left\{x \in X: d\left(x, x_{2}\right) \geq d\left(x_{1}, x_{2}\right)\right\}$.

Theorem 1. Let $T: A(X, E) \rightarrow A(Y, F)$ be a Riesz isomorphism preserving nonvanishing functions. Then $T$ carries the form

$$
(T f)(y)=(J y) f(\varphi(y)), \quad \forall f \in A(X, E), y \in Y .
$$

Here, $\varphi$ is a homeomorphism from $Y$ onto $X$ and all fiber linear maps Jy: E $\rightarrow$ F are isomorphisms.

Remark 2. When $\alpha=1$, the previous theorem is not valid for the little Lipschitz space $\operatorname{lip}_{1}(X, E)$, where $X$ is a connected Banach and $E$ is a Banach lattice. Note that if $X$ is a connected Banach spaces, we have that $\operatorname{lip}_{1}^{b}(X, E)=\operatorname{lip}_{1}(X, E)$ consisting of all $E$-valued constant functions defined on $X$. Let $\varphi$ be any map from $\mathbb{R}^{2}$ to $\mathbb{R}$ and $T: \operatorname{lip}_{1}(\mathbb{R}, E) \rightarrow \operatorname{lip}_{1}\left(\mathbb{R}^{2}, E\right)$ a linear bijection operator defined by

$$
T f(y)=f(\varphi(y)), \quad \forall y \in \mathbb{R}^{2} .
$$

It is obvious that the operator $T$ is a Riesz isomorphism preserving nonvanishing functions with a weighted composition representation, but $\mathbb{R}$ and $\mathbb{R}^{2}$ are not homeomorphic.

It is easy to prove the following lemma.

Lemma 3. T preserves common zeros, that is,

$$
\bigcap_{i=1}^{n} z\left(f_{i}\right) \neq \emptyset \Longleftrightarrow \bigcap_{i=1}^{n} z\left(T f_{i}\right) \neq \emptyset
$$

for any $f_{1}, \ldots, f_{n} \in A(X, E)$ and $n \in \mathbb{N}$.

Proof of Theorem 1. In the above contexts, $A(X, E)$ and $A(Y, F)$ contain constant functions, so $E_{y}=E$ and $F_{y}=F$, where $E_{y}, F_{y}$ are defined in [14, Definition 3.8]. Therefore, by [14, Theorem 3.1] we can derive the result.

Lemma 4. In the Contexts 1 and 2, $T$ is automatically continuous. 
Proof. We are going to use the Closed Graph Theorem to prove this lemma. Suppose that the sequence of functions $\left\{f_{n}\right\}$ converges to $f_{0}$ in $A(X, E)$ and $\left\{T f_{n}\right\}$ converges to $g_{0}$ in $A(Y, F)$; then for any $x \in X$ and $y \in Y$, we have that $\left\{f_{n}(x)\right\}$ converges to $f_{0}(x)$ in $E$ and $\left\{\left(T f_{n}\right)(y)\right\}$ converges to $g_{0}(y)$ in $F$, respectively. Notice that, for any $x \in X, J \varphi^{-1}(x): E \rightarrow F$ is continuous; then one can derive that

$$
\begin{aligned}
\left(T f_{n}\right)\left(\varphi^{-1}(x)\right) & =\left(J \varphi^{-1}(x)\right) f_{n}(x) \longrightarrow\left(J \varphi^{-1}(x)\right) f_{0}(x) \\
& =\left(T f_{0}\right)\left(\varphi^{-1}(x)\right)
\end{aligned}
$$

for all $x$ in $X$. Since $\varphi$ is a bijection from $Y$ onto $X$, we get that the sequence $\left\{\left(T f_{n}\right)(y)\right\}$ converges to $\left(T f_{0}\right)(y)$ for all $y$ in $Y$, and hence $g_{0}=T f_{0}$. This means that $T$ is a closed operator from $A(X, E)$ to $A(Y, F)$, and then $T$ is continuous.

In order to prove that $\varphi$ is a $\alpha$-Lipschitz map from $Y$ onto $X$, we need the following lemma, and some idea of the proof comes from [15, Lemma 5.8].

Lemma 5. For any fixed element $e \in E$ with $\|e\|=1$, we have that

$$
\inf _{y \in Y}\|(J y)(e)\|=\inf _{y \in Y}\|T(1 \otimes e)(y)\|>0 .
$$

Proof. By Theorem 1 we can also find a map $\widetilde{J}$ from $X$ to $\operatorname{Iso}(F, E)$ (which is the set of all linear isomorphisms from $F$ to $E$ ) and a bijection $\tilde{\varphi}$ from $X$ onto $Y$ such that

$$
\left(T^{-1} g\right)(x)=(\widetilde{J} x) g(\widetilde{\varphi}(x))
$$

for all $x \in X$ and $g \in A(Y, F)$. From the definition of $\psi, \varphi$, and $\widetilde{\varphi}$, we can see that $\varphi^{-1}=\widetilde{\varphi}=\psi$.

Suppose on the contrary that there exists a sequence $\left\{y_{n}\right\} \subset Y$ such that $\left\|T(1 \otimes e)\left(y_{n}\right)\right\|=\left\|\left(J y_{n}\right)(e)\right\| \leq 2^{-2 n}$ for all $n \in \mathbb{N}$. If $\left\{y_{n}\right\}$ has a limit point $y^{\prime}$ in $Y$, notice that $T$ preserves nonvanishing functions, then we can see that $\left(J y^{\prime}\right)(e)=0$ and hence $e=0$. This leads to a contradiction. On the other hand, if there exists a positive scalar $\tau>0$ such that $d^{\alpha}\left(y_{n}, y_{m}\right) \geq \tau$ for any $n, m \in \mathbb{N}$ with $n \neq m$, when we take the norm one element

$$
b_{n}=\frac{T(1 \otimes e)\left(y_{n}\right)}{\left\|T(1 \otimes e)\left(y_{n}\right)\right\|},
$$

then we can derive that

$$
\begin{gathered}
{\left[T^{-1}(T(1 \otimes e))\right]\left(\varphi\left(y_{n}\right)\right)=(1 \otimes e)\left(\varphi\left(y_{n}\right)\right)=e,} \\
{\left[T^{-1}(T(1 \otimes e))\right]\left(\varphi\left(y_{n}\right)\right)=\left(\widetilde{J} \varphi\left(y_{n}\right)\right) T(1 \otimes e)\left(y_{n}\right)}
\end{gathered}
$$

for all $n \in \mathbb{N}$. Therefore, for any $n \in \mathbb{N}$, we know that

$$
\begin{aligned}
\left\|\left(\widetilde{J} \varphi\left(y_{n}\right)\right)\left(b_{n}\right)\right\| & =\frac{\left\|\left(\tilde{J} \varphi\left(y_{n}\right)\right) T(1 \otimes e)\left(y_{n}\right)\right\|}{\left\|T(1 \otimes e)\left(y_{n}\right)\right\|} \\
& =\frac{1}{\left\|T(1 \otimes e)\left(y_{n}\right)\right\|} \geq 2^{2 n} .
\end{aligned}
$$

Moreover, for any $n \in \mathbb{N}$, by the similar manner of (7) and (8) we can define the function $\psi_{n}(y) \in A^{b}(Y)$ such that $0 \leq \psi_{n} \leq$ $1, L_{\alpha}\left(\psi_{n}\right) \leq m$ for some $m>0, \psi_{n}\left(y_{n}\right)=1$ and $\psi_{n}(y)=0$ for all $y$ such that $d\left(y, y_{n}\right) \geq \tau / 2$. When put

$$
h_{0}=\sum_{n=2}^{\infty} \frac{\psi_{n} \otimes b_{n}}{2^{n}}
$$

we can see that $h_{0}$ belongs to $A^{b}(Y, F)$ and $h_{0}\left(y_{n}\right)=b_{n} / 2^{n}$ for $n>1$. Then one can conclude that

$$
\begin{aligned}
\left(T^{-1} h_{0}\right)\left(\varphi\left(y_{n}\right)\right) & =\left(\widetilde{J} \varphi\left(y_{n}\right)\right)\left(h_{0}\left[\widetilde{\varphi}\left(\varphi\left(y_{n}\right)\right)\right]\right) \\
& =\left(\widetilde{J} \varphi\left(y_{n}\right)\right)\left(h_{0}\left(y_{n}\right)\right)=\frac{1}{2^{n}}\left(\widetilde{J} \varphi\left(y_{n}\right)\right)\left(b_{n}\right),
\end{aligned}
$$

and hence

$$
\left\|\left(T^{-1} h_{0}\right)\left(\varphi\left(y_{n}\right)\right)\right\|=\frac{\left\|\left(\widetilde{J} \varphi\left(y_{n}\right)\right)\left(b_{n}\right)\right\|}{2^{n}}>2^{n}, \quad \forall n \geq 2 .
$$

This is a contradiction in Contexts 1 and 2 since $\left\|T^{-1} h_{0}\right\|_{\infty}<$ $\infty$.

Theorem 6. Suppose that $X, Y$ are bounded metric spaces in the Contexts 1 and 2; then $\varphi$ is a $\alpha$-Lipschitz map from $Y$ onto $X$.

Proof. We can define the linear map $\widetilde{T}$ from $A(X)$ to $A(Y)$ by

$$
(\widetilde{T} f)(y)=f(\varphi(y)), \quad \forall y \in Y \text {. }
$$

We have to show that $\widetilde{T}$ is well defined at first. For any fixed element $e \in E$ with $\|e\|=1$, from Lemma 5 we can choose a positive scalar $v$ such that $\|T(1 \otimes e)(y)\| \geq v>0$ for all $y$ in $Y$, and then it is easy to see that the function $h$ which maps $y$ to $1 /\|T(1 \otimes e)(y)\|$ belongs to $A(Y)$.

Assume that $f$ is a positive function in $A(X)$; one can get that, for any $y_{1}, y_{2} \in Y$,

$$
\begin{aligned}
\mid(\widetilde{T} f) & \left(y_{1}\right)\left\|T(1 \otimes e)\left(y_{1}\right)\right\|-(\widetilde{T} f)\left(y_{2}\right)\left\|T(1 \otimes e)\left(y_{2}\right)\right\| \mid \\
= & \left\|\left(J y_{1}\right) f\left(\varphi\left(y_{1}\right)\right) e\right\|-\left\|\left(J y_{2}\right) f\left(\varphi\left(y_{2}\right)\right) e\right\| \mid \\
\leq & \left\|\left(J y_{1}\right) f\left(\varphi\left(y_{1}\right)\right) e-\left(J y_{2}\right) f\left(\varphi\left(y_{2}\right)\right) e\right\| \\
= & \left\|T(f \otimes e)\left(y_{1}\right)-T(f \otimes e)\left(y_{2}\right)\right\| \\
\leq & L_{\alpha}(T(f \otimes e)) d^{\alpha}\left(y_{1}, y_{2}\right) \leq\|T(f \otimes e)\| d^{\alpha}\left(y_{1}, y_{2}\right) ;
\end{aligned}
$$

that is, $(\widetilde{T} f)(y)\|T(1 \otimes e)(y)\|$ is a bounded $\alpha$-Lipschitz function. Moreover, in Context 2 we can derive that $(\widetilde{T} f)(y) \| T(1 \otimes$ $e)(y) \|$ is also a little Lipschitz function. This means that the function $(\widetilde{T} f)(y)=(\widetilde{T} f)(y)\|T(1 \otimes e)(y)\| h(y)$ belongs to $A(Y)$. Therefore, $\widetilde{T}$ is a well-defined bijective linear operator from $A(X)$ onto $A(Y)$, and $\widetilde{T}$ is also a nonvanishing preserver. 
Suppose that $\left\{f_{n}\right\}$ is a sequence which converges to 0 in $A(X)$ and the sequence $\left\{\widetilde{T} f_{n}\right\}$ converges to $g_{0}$ in $A(Y)$. For any $n \in \mathbb{N}$ and $y \in Y,\left(\widetilde{T} f_{n}\right)(y)=f_{n}(\varphi(y))$, and hence we have that $\left\{f_{n}(\varphi(y))\right\}$ converges to $g_{0}(y)$ for all $y \in Y$. Notice that $\left\{f_{n}\right\}$ converges to 0 ; one can conclude that $\left\{f_{n}(x)\right\}$ converges to 0 for all $x \in X$, and, since $\varphi$ is a bijective map from $Y$ onto $X$, we have that $g_{0}(y)=0$ for any $y$ in $Y$. Therefore, $\widetilde{T}$ is a closed operator and hence $\widetilde{T}$ is continuous.

For any $y_{1}$ and $y_{2}$ in $Y$, there exists a function $f_{0} \in$ $A(X)$ such that $\left\|f_{0}\right\| \leq D(X)+D(X)^{1-\alpha}$ and $f_{0}\left(\varphi\left(y_{1}\right)\right)=$ $d\left(\varphi\left(y_{1}\right), \varphi\left(y_{2}\right)\right)$ and $f_{0}\left(\varphi\left(y_{2}\right)\right)=0$ (in fact, $f_{0}(x)=$ $d\left(x, \varphi\left(y_{2}\right)\right)$ has the properties that we need). Here $D(X)$ denotes the diameter of $X$. Then we can derive that

$$
\begin{aligned}
\left|\left(\widetilde{T} f_{0}\right)\left(y_{1}\right)-\left(\widetilde{T} f_{0}\right)\left(y_{2}\right)\right| & \leq L_{\alpha}\left(\widetilde{T} f_{0}\right) d^{\alpha}\left(y_{1}, y_{2}\right) \\
& \leq\|\widetilde{T}\|\left\|f_{0}\right\| d^{\alpha}\left(y_{1}, y_{2}\right) .
\end{aligned}
$$

Furthermore, we have that

$$
\begin{aligned}
d\left(\varphi\left(y_{1}\right), \varphi\left(y_{2}\right)\right) & =\left|f_{0}\left(\varphi\left(y_{1}\right)\right)-f_{0}\left(\varphi\left(y_{2}\right)\right)\right| \\
& =\left|\left(\widetilde{T} f_{0}\right)\left(y_{1}\right)-\left(\widetilde{T} f_{0}\right)\left(y_{2}\right)\right| \\
& \leq\|\widetilde{T}\|\left(D(X)+D(X)^{1-\alpha}\right) d^{\alpha}\left(y_{1}, y_{2}\right),
\end{aligned}
$$

and this means that $\varphi$ is a $\alpha$-Lipschitz mapping from $Y$ onto $X$. Similarly, we can see that $\varphi^{-1}$ is also $\alpha$-Lipschitz, and then $\varphi$ is a $\alpha$-Lipschitz homeomorphism.

For the spaces of scalar-valued Lipschitz functions, we give a complete characterization of nonvanishing preservers. But at first we need to recall a special case of [16, Lemma 25].

Lemma 7. Let $A(X), A(Y)$ be in Contexts 1 and 2. Suppose that $T: A(X) \rightarrow A(Y)$ is a linear nonvanishing preserver; then the map $S: A(X) \rightarrow A(Y)$ given by

$$
S f=T f \cdot \frac{T 1}{|T 1|}
$$

is a Riesz isomorphism preserving nonvanishing functions.

Proof. For completeness, we will sketch the proof. Observe that $T 1$ is never vanishing. If $f \in A(X)$ and $\lambda \in \mathbb{R}$, then $\lambda \in \operatorname{range} f$ if and only if $0 \in \operatorname{range}(f-\lambda)$ if and only if $0 \in$ range $(T f-\lambda T 1)$ if and only if $\lambda \in$ range $T f / T 1$. In particular, if $f \geq 0$, then $T f / T 1 \geq 0$. Let $Y_{+}=\{y \in Y:(T 1)(y)>0\}$ and $Y_{-}=\{y \in Y:(T 1)(y)<0\}$. Then $Y_{+} \cup Y_{-}$is a partition of $Y$ into two open sets.

Suppose that $f \in A(X)$ and $f \geq 0$. Then $T f \geq 0$ on $Y_{+}$ and $T f \leq 0$ on $Y_{-}$. Hence $T f \cdot T 1 /|T 1|=|T f| \in A(Y)$. For any $f \in A(X)$, we have that $f_{+}, f_{-} \in A(X)$, and $\left|T\left(f_{+}\right)\right|=$ $T\left(f_{+}\right) \cdot T 1 /|T 1|$ and $\left|T\left(f_{-}\right)\right|=T\left(f_{-}\right) \cdot T 1 /|T 1|$. Then we can derive that

$$
\begin{aligned}
S f & =T f \cdot \frac{T 1}{|T 1|}=\left(T\left(f_{+}\right)-T\left(f_{-}\right)\right) \cdot \frac{T 1}{|T 1|} \\
& =\left|T\left(f_{+}\right)\right|-\left|T\left(f_{-}\right)\right| \in A(Y) .
\end{aligned}
$$

This means that $S$ is well defined. Moreover, it is easy to check that $S$ is bijective.

From the previous paragraph, if $0 \leq f \in A(X)$, then $S f=$ $|T f| \geq 0$. If $f \in A(X)$ and $g=S f \geq 0$, then by the above,

$$
0 \leq S f=T f \cdot \frac{T 1_{X}}{\left|T 1_{X}\right|}=\left|T\left(f_{+}\right)\right|-\left|T\left(f_{-}\right)\right| .
$$

By [17, Lemma 2.3], $T$ is biseparating, and hence $T\left(f_{+}\right)$. $T\left(f_{-}\right)=0$. It follows that $T\left(f_{-}\right)=0$ and thus $f_{-}=0$. Therefore, $f \geq 0$. Thus $S$ is a Riesz isomorphism. It is trivial to check that $0 \in$ range $f$ if $0 \in$ range $S f$ for any $f \in A(X)$.

Theorem 8. Suppose that $X, Y$ are bounded metric spaces and $T$ is a nonvanishing preserver between the following function spaces:

(i) $0<\alpha \leq 1$ and $T: \operatorname{Lip}_{\alpha}(X) \rightarrow \operatorname{Lip}_{\alpha}(Y)$;

(ii) $0<\alpha<1$ and $T: \operatorname{Lip}_{\alpha}(X) \rightarrow \operatorname{Lip}_{\alpha}(Y)$.

Then $T$ is a weighted composition operator of the form

$$
(T f)(y)=h(y) f(\varphi(y)) .
$$

Here $h=T 1$ and $\varphi: Y \rightarrow X$ is a $\alpha$-Lipschitz map.

Proof. By Lemma 7 we have that $T$ is a Riesz isomorphism. Then by Theorem 6 we can derive the conclusion.

In Theorem 8, the boundedness of the metric spaces can not be dropped.

Example 9. Let $\mathbb{N}_{1}$ be the positive integers with the discrete metric, and we can derive that $\mathbb{N}_{1}$ is not Lipchitz homeomorphic to $\mathbb{N}$. By [18, Example 1.6.4] we can derive that $\operatorname{Lip}^{b}(\mathbb{N})=\operatorname{Lip}^{b}\left(\mathbb{N}_{1}\right)=\ell^{\infty}$, and then the identity map $I$ : $\operatorname{Lip}^{b}(\mathbb{N}) \rightarrow \operatorname{Lip}^{b}\left(\mathbb{N}_{1}\right)$ is a nonvanishing preserver. However, the underlying metric spaces are not Lipschitz homeomorphic.

\section{Compact Weighted Composition Operators on Lipschitz Spaces}

Suppose that $X, Y$ are metric spaces, $0<\alpha \leq 1$, and $T$ : $\operatorname{Lip}_{\alpha}^{b}(X) \rightarrow \operatorname{Lip}_{\alpha}^{b}(Y)$ is a weighted composition operator, that is,

$$
(T f)(y)=h(y) f(\varphi(y)), \quad \forall y \in Y, f \in \operatorname{Lip}_{\alpha}^{b}(X) .
$$

Here $h=T 1$ and $\varphi: Y \rightarrow X$ is a $\alpha$-Lipschitz mapping. Put $Y_{0}=\{y \in Y: h(y)=0\}$. Recall that $\varphi: Y \rightarrow X$ is supercontractive on $Y^{\prime} \subset Y$ if for each $\varepsilon>0$ there exists $\delta>0$ such that $d\left(\varphi\left(y_{1}\right), \varphi\left(y_{2}\right)\right)<\varepsilon d\left(y_{1}, y_{2}\right)$ whenever $y_{1}, y_{2} \in Y^{\prime}$ and $0<d\left(y_{1}, y_{2}\right)<\delta$. In this section, we will characterize the compact weighted composition operator $T$ and consider its spectrum.

Theorem 10. Suppose that $T$ is compact. For any $y_{0} \in Y \backslash$ $Y_{0}$, there is an open neighborhood $U_{0}$ of $y_{0}$ such that $\varphi$ is supercontractive on $U_{0}$ and $\varphi\left(U_{0}\right)$ is totally bounded. 
Proof. Since $h\left(y_{0}\right) \neq 0$, we can find an open neighborhood $U_{0}$ of $y_{0}$ such that $|h(y)| \geq\left|h\left(y_{0}\right)\right| / 2>0$ for all $y \in U_{0}$. Suppose on the contrary that there exist $\left\{x_{n}\right\},\left\{y_{n}\right\} \subset U_{0}$ such that $d\left(x_{n}, y_{n}\right) \rightarrow 0$ and

$$
\frac{d\left(\varphi\left(x_{n}\right), \varphi\left(y_{n}\right)\right)}{d\left(x_{n}, y_{n}\right)}>\varepsilon_{0}
$$

for some $\varepsilon_{0}>0$. Without loss of generality we can assume that $d^{\alpha^{2}}\left(x_{n}, y_{n}\right)<1 / n$.

Let

$$
f_{n}(x)=\frac{1-e^{-n d^{\alpha}\left(x, \varphi\left(y_{n}\right)\right)}}{n} ;
$$

we can derive that $\left\|f_{n}\right\|_{\infty} \leq 1 / n$ and $\left|f_{n}\left(x_{1}\right)-f_{n}\left(x_{2}\right)\right| \leq$ $d^{\alpha}\left(x_{1}, x_{2}\right)$ for any $x_{1}, x_{2} \in X$. This implies that $\left\{f_{n}\right\}$ is a bounded sequence in $\operatorname{Lip}_{\alpha}^{b}(X)$. If $T$ is compact, then there exists a subsequence $\left\{f_{n_{k}}\right\}$ such that $T f_{n_{k}} \rightarrow g_{0} \in \operatorname{Lip}_{\alpha}^{b}(Y)$. Since $f_{n} \rightarrow 0$ uniformly, for any $y \in Y$, we have that

$$
\left|\left(T f_{n_{k}}\right)(y)\right|=\left|h(y) f_{n_{k}}(\varphi(y))\right| \leq\|h\|_{\infty}\left|f_{n_{k}}(\varphi(y))\right| \longrightarrow 0,
$$

and then $g_{0}=0$. This means that $T f_{n_{k}} \rightarrow 0$ in $\operatorname{Lip}_{\alpha}^{b}(Y)$.

On the other hand, for any $n \in \mathbb{N}$, by the Mean Value Theorem we have that

$$
\begin{aligned}
\frac{\left|\left(T f_{n}\right)\left(x_{n}\right)-\left(T f_{n}\right)\left(y_{n}\right)\right|}{d^{\alpha}\left(x_{n}, y_{n}\right)} & =\frac{\left|h\left(x_{n}\right) f_{n}\left(\varphi\left(x_{n}\right)\right)\right|}{d^{\alpha}\left(x_{n}, y_{n}\right)} \\
& \geq \frac{\left|h\left(y_{0}\right)\right|}{2} \frac{1-e^{-n d^{\alpha}\left(\varphi\left(x_{n}\right), \varphi\left(y_{n}\right)\right)}}{n d^{\alpha}\left(x_{n}, y_{n}\right)} \\
& =\frac{\left|h\left(y_{0}\right)\right|}{2} e^{-n \xi_{n}} \frac{d^{\alpha}\left(\varphi\left(x_{n}\right), \varphi\left(y_{n}\right)\right)}{d^{\alpha}\left(x_{n}, y_{n}\right)} \\
& \geq \frac{\left|h\left(y_{0}\right)\right|}{2} e^{-L_{\alpha}^{\alpha}(\varphi)} \varepsilon_{0}^{\alpha} .
\end{aligned}
$$

Here $0<\xi_{n}<d^{\alpha}\left(\varphi\left(x_{n}\right), \varphi\left(y_{n}\right)\right) \leq L_{\alpha}^{\alpha}(\varphi) d^{\alpha^{2}}\left(x_{n}, y_{n}\right)<$ $L_{\alpha}^{\alpha}(\varphi) / n$. Therefore, we can derive that $L_{\alpha}\left(T f_{n}\right) \nrightarrow 0$, and this is a contradiction.

On the other hand, suppose on the contrary that $\varphi\left(U_{0}\right)$ is not totally bounded, then there exist a constant $\tau>0$ and $z_{n}=\varphi\left(u_{n}\right) \in \varphi\left(U_{0}\right)$ such that $d^{\alpha}\left(z_{n}, z_{m}\right)>\tau$ whenever $n \neq m$. Let

$$
f_{n}(x)=1-e^{-d^{\alpha}\left(x, z_{n}\right)}, \quad \forall x \in X ;
$$

then it is easy to see that $f_{n}\left(z_{n}\right)=0$ and $\left\|f_{n}\right\| \leq 1$. Moreover, for any $n \neq m$, we can derive that

$$
\begin{aligned}
\left\|T f_{n}-T f_{m}\right\|_{\infty} & \geq\left|h\left(u_{n}\right) f_{m}\left(z_{n}\right)\right| \\
& \geq \frac{\left|h\left(y_{0}\right)\right|}{2}\left(1-e^{-d^{\alpha}\left(z_{n}, z_{m}\right)}\right) \\
& \geq \frac{\left|h\left(y_{0}\right)\right|}{2}\left(1-e^{-\tau}\right) .
\end{aligned}
$$

Therefore, $\left\{T f_{n}\right\}$ has no Cauchy subsequence, and hence $T$ is not compact. This leads to a contradiction.

Theorem 11. Suppose that $\varphi$ is supercontractive on $Y \backslash Y_{0}$ and $\varphi\left(Y \backslash Y_{0}\right)$ is totally bounded; then the weighted composition operator defined by (29) is compact.

Proof. Let $\left\{f_{n}\right\} \subset \operatorname{Lip}_{\alpha}^{b}(X)$ be a bounded sequence, that is, $\left\|f_{n}\right\| \leq M$ for some $M>0$. Since $\varphi\left(Y \backslash Y_{0}\right)$ is totally bounded, there exists a subsequence of $\left\{f_{n}\right\}$, which is also denoted by $\left\{f_{n}\right\}$, such that $\left\{f_{n}\right\}$ is convergent uniformly in $\varphi\left(Y \backslash Y_{0}\right)$. Denote the limit by $f_{0}(x)$ for all $x \in \varphi\left(Y \backslash Y_{0}\right)$. It is easy to verify that $f_{0}$ is a bounded Lipschitz function in $\varphi\left(Y \backslash Y_{0}\right)$. By the similar argument of [18, Theorem 1.5.6] we can extend $f_{0}$ to be a bounded Lipschitz function in $\operatorname{Lip}_{\alpha}^{b}(X)$, which is also denoted by $f_{0}$. It suffices to show that $\left\{T f_{n}\right\}$ converges to $T f_{0}$ in $\operatorname{Lip}_{\alpha}^{b}(Y)$.

Since $T$ is a weighted composition operator, it is easy to see that $\left\{T f_{n}\right\}$ converges to $T f_{0}$ uniformly on $Y$. Let $\varepsilon>0$ be given. Since $\varphi$ is supercontractive on $Y \backslash Y_{0}$, there exists $\delta>0$ such that

$$
\frac{d\left(\varphi\left(y_{1}\right), \varphi\left(y_{2}\right)\right)}{d\left(y_{1}, y_{2}\right)}<\varepsilon
$$

whenever $y_{1}, y_{2} \in Y \backslash Y_{0}$ and $0<d\left(y_{1}, y_{2}\right)<\delta$.

We will show that $L_{\alpha}\left(T f_{n}-T f_{0}\right) \rightarrow 0$ by dividing into four cases as the following arguments. For any $y_{1}, y_{2} \in Y$ with $y_{1} \neq y_{2}$.

Case 1. If $y_{1}, y_{2} \in Y_{0}$, we have that $\left(T f_{n}\right)\left(y_{i}\right)=\left(T f_{0}\right)\left(y_{i}\right)=0$ for $i=1,2$.

Case 2. If $y_{1}, y_{2} \in Y \backslash Y_{0}$ and $0<d\left(y_{1}, y_{2}\right)<\delta$, we have that

$$
\begin{aligned}
\left|T\left(f_{n}-f_{0}\right)\left(y_{1}\right)-T\left(f_{n}-f_{0}\right)\left(y_{2}\right)\right| \\
\leq||\left[h\left(y_{1}\right)-h\left(y_{2}\right)\right]\left(f_{n}-f_{0}\right)\left(\varphi\left(y_{1}\right)\right) \mid \\
\quad+\left|h\left(y_{2}\right)\left[\left(f_{n}-f_{0}\right)\left(\varphi\left(y_{1}\right)\right)-\left(f_{n}-f_{0}\right)\left(\varphi\left(y_{2}\right)\right)\right]\right| \\
\leq L_{\alpha}(h) d^{\alpha}\left(y_{1}, y_{2}\right)\left|\left(f_{n}-f_{0}\right)\left(\varphi\left(y_{1}\right)\right)\right| \\
\quad+\|h\|_{\infty}\left(L_{\alpha}\left(f_{n}\right)+L_{\alpha}\left(f_{0}\right)\right) d^{\alpha}\left(\varphi\left(y_{1}\right), \varphi\left(y_{2}\right)\right) .
\end{aligned}
$$

Moreover, by (36) we can derive that

$$
\begin{aligned}
d^{\alpha}\left(\varphi\left(y_{1}\right), \varphi\left(y_{2}\right)\right) & =\frac{d^{\alpha}\left(\varphi\left(y_{1}\right), \varphi\left(y_{2}\right)\right)}{d^{\alpha}\left(y_{1}, y_{2}\right)} d^{\alpha}\left(y_{1}, y_{2}\right) \\
& \leq \varepsilon^{\alpha} d^{\alpha}\left(y_{1}, y_{2}\right) .
\end{aligned}
$$

Case 3. If $y_{1}, y_{2} \in Y \backslash Y_{0}$ and $d\left(y_{1}, y_{2}\right)>\delta$, we have that

$$
\begin{gathered}
\frac{\left|T\left(f_{n}-f_{0}\right)\left(y_{1}\right)-T\left(f_{n}-f_{0}\right)\left(y_{2}\right)\right|}{d^{\alpha}\left(y_{1}, y_{2}\right)} \\
\leq \frac{2\left\|T f_{n}-T f_{0}\right\|_{\infty}}{\delta^{\alpha}} .
\end{gathered}
$$


Case 4. If $y_{1} \in Y \backslash Y_{0}$ and $y_{2} \in Y_{0}$, we have that $h\left(y_{2}\right)=0$ and then

$$
\begin{aligned}
\left|T\left(f_{n}-f_{0}\right)\left(y_{1}\right)-T\left(f_{n}-f_{0}\right)\left(y_{2}\right)\right| \\
\quad=\left|h\left(y_{1}\right)\left(f_{n}-f_{0}\right)\left(\varphi\left(y_{1}\right)\right)\right| \\
\quad=\left|h\left(y_{1}\right)-h\left(y_{2}\right)\right| \cdot\left|\left(f_{n}-f_{0}\right)\left(\varphi\left(y_{1}\right)\right)\right| \\
\quad \leq L_{\alpha}(h) d^{\alpha}\left(y_{1}, y_{2}\right)\left|\left(f_{n}-f_{0}\right)\left(\varphi\left(y_{1}\right)\right)\right| .
\end{aligned}
$$

Hence we derive that $L_{\alpha}\left(T f_{n}-T f_{0}\right) \rightarrow 0$ and then $T f_{n} \rightarrow$ $T f_{0}$. This means that $T$ is a compact operator.

By the similar argument, one can conclude the following results for the scalar-valued little Lipschitz function spaces.

Theorem 12. Let $\alpha \in(0,1)$. Suppose that $T: \operatorname{Lip}_{\alpha}^{b}(X) \rightarrow$ $L_{i p}^{b}(Y)$ is a nonzero weighted composition operator of the form (29).

(1) If $T$ is compact, then, for any $y_{0} \in Y \backslash Y_{0}$, there is an open neighborhood $U_{0}$ of $y_{0}$ such that $\varphi$ is supercontractive on $U_{0}$ and $\varphi\left(U_{0}\right)$ is totally bounded.

(2) If $\varphi$ is supercontractive on $Y \backslash Y_{0}$ and $\varphi\left(Y \backslash Y_{0}\right)$ is totally bounded, then $T$ is compact.

Also here, the result of [19] also refers to the case where $T$ is a composition operator.

Corollary 13. Suppose that $X, Y$ are compact metric spaces, and $T$ is a weighted composition operator of the form (29) between the following function spaces:

(i) $0<\alpha \leq 1$ and $T: \operatorname{Lip}_{\alpha}^{b}(X) \rightarrow \operatorname{Lip}_{\alpha}^{b}(Y)$;

(ii) $0<\alpha<1$ and $T: \operatorname{Lip}_{\alpha}^{b}(X) \rightarrow \operatorname{Lip}_{\alpha}^{b}(Y)$.

Then $T$ is compact if and only if $\varphi$ is supercontractive on $Y \backslash Y_{0}$.

When $T$ is a composition operator, that is, $h=T 1=1$ in the form (29), then $Y_{0}=\emptyset$ and we can establish the following results in [20, Theorem 1.1].

Corollary 14. Suppose that $X, Y$ are metric spaces and $T$ : $\operatorname{Lip}_{\alpha}^{b}(X) \rightarrow \operatorname{Lip}_{\alpha}^{b}(Y)$ is a composition operator; then $T$ is compact if and only if $\varphi$ is supercontractive and $\varphi(Y)$ is totally bounded.

In the following part of this section we have $X=Y$. Define $\varphi_{0}(x)=x$ and $\varphi_{n}(x)=\varphi\left(\varphi_{n-1}(x)\right)$ for all $x \in X$ by induction. A point $x_{0} \in X$ is said to be the fixed point of $\varphi$ of order $n$, $n \in \mathbb{N}$, if $\varphi_{n}\left(x_{0}\right)=x_{0}$ and $\varphi_{i}\left(x_{0}\right) \neq x_{0}$ for any $i=0,1, \ldots, n-1$.

Theorem 15. Let $X$ be a complete metric space and $T$ : $\operatorname{Lip}^{b}(X) \rightarrow \operatorname{Lip}^{b}(X)$ a weighted composition operator of form (29) satisfying: $\varphi$ is supercontractive on $X \backslash X_{0}$ and $\varphi\left(X \backslash X_{0}\right)$ is totally bounded. Then we can derive that $\sigma(T)=\{0\} \cup \mathcal{S}$, where

$$
\mathcal{S}=\left\{\lambda: \lambda^{n}=h\left(x_{0}\right) h\left(\varphi\left(x_{0}\right)\right) \cdots h\left(\varphi_{n-1}\left(x_{0}\right)\right),\right.
$$

$x_{0}$ is a fixed point of $\varphi$ of order $\left.n\right\}$.
Proof. Suppose that $x_{0}$ is a fixed point of $\varphi$ of order $n$. If $h\left(\varphi_{k}\left(x_{0}\right)\right)=0$ for some $k$, we can see that $T$ is not surjective and hence $0 \in \sigma(T)$.

Assume that $h\left(\varphi_{k}\left(x_{0}\right)\right) \neq 0$ for any $k=0,1,2, \ldots, n-1$ and $\lambda^{n}=h\left(x_{0}\right) \cdots h\left(\varphi_{n-1}\left(x_{0}\right)\right)$.

When $n=1$, we have that $\lambda=h\left(x_{0}\right)$ and $\varphi\left(x_{0}\right)=x_{0}$. There exists $g \in \operatorname{Lip}^{b}(X)$ such that $g\left(x_{0}\right)=1$. There is no $f \in \operatorname{Lip}^{b}(X)$ such that $(\lambda-T) f=g$. Indeed, if such $f$ exists, we can derive that

$$
\begin{aligned}
0 & =\lambda f\left(x_{0}\right)-h\left(x_{0}\right) f\left(x_{0}\right)=\lambda f\left(x_{0}\right)-h\left(x_{0}\right) f\left(\varphi\left(x_{0}\right)\right) \\
& =g\left(x_{0}\right)=1,
\end{aligned}
$$

and this is impossible. This means that $\lambda \in \sigma(T)$.

When $n \geq 2$, let $\delta:=\min \left\{d\left(\varphi_{i}\left(x_{0}\right), \varphi_{j}\left(x_{0}\right)\right): 0 \leq i \neq j \leq\right.$ $n-1\}$, and define

$$
\begin{aligned}
g=\frac{1}{n} \sum_{i=0}^{n-1} & \frac{1}{\lambda^{n-i-1} h\left(x_{0}\right) \cdots h\left(\varphi_{i-1}\left(x_{0}\right)\right)} \\
& \times \max \left\{0,1-\frac{d\left(x, \varphi_{i}\left(x_{0}\right)\right)}{\delta}\right\} .
\end{aligned}
$$

Here $h\left(\varphi_{-1}\left(x_{0}\right)\right):=1$. Then, similar to the argument of [3, Proposition 3], we can derive that $\lambda \in \sigma(T)$.

On the other hand, for each $f \in \operatorname{Lip}^{b}(X)$ with $\lambda f=T f$, for some $\lambda \notin\{0\} \cup \mathcal{S}$, we will prove that $f=0$. This implies that $\lambda \notin \sigma(T)$ and completes the proof.

From the assumption $\lambda f=T f=h \cdot f \circ \varphi$, for all $x \in X$ and $n \in \mathbb{N}$, we derive that

$$
\lambda^{n} f(x)=h(x) h(\varphi(x)) \cdots h\left(\varphi_{n-1}(x)\right) f\left(\varphi_{n}(x)\right) .
$$

Given any $z \in X$, let $\mathscr{F}=\left\{\varphi_{n}(z): n \in \mathbb{N} \cup\{0\}\right\}$ and $\mathcal{N}=\left\{n \in \mathbb{N}:\left|h\left(\varphi_{n}(z)\right)\right| \geq \delta_{0}\right\}$; here $\delta_{0}$ is any fixed number with $0<\delta_{0}<|\lambda|$. We provide that $f(z)=0$, which implies that $f=0$, by dividing into the following cases.

Case $I\left(\mathscr{F} \cap X_{0} \neq \emptyset\right)$. If there exists $i_{0}$ such that $h\left(\varphi_{i_{0}}(z)\right)=0$, by (44) we can see that

$$
\lambda^{i_{0}+1} f(z)=h(z) h(\varphi(z)) \cdots h\left(\varphi_{i_{0}}(z)\right) f\left(\varphi_{i_{0}+1}(z)\right)=0 .
$$

This implies that $f(z)=0$.

Case II ( $\mathscr{F} \subset X \backslash X_{0}$ and $\mathscr{F}$ is finite). Let $\mathscr{F}=$ $\left\{z, \varphi(z), \ldots, \varphi_{n_{0}}(z)\right\}$. Then there exists $0 \leq k \leq n_{0}$ such that $\varphi_{n_{0}+1}(z)=\varphi_{k}(z)$. This means that $\varphi_{k}(z)$ is a fixed point of $\varphi$ of order $n_{0}-k+1$. By (44), we have that

$$
\begin{array}{rl}
\lambda^{n_{0}-k+1} & f\left(\varphi_{k}(z)\right) \\
& =h\left(\varphi_{k}(z)\right) h\left(\varphi_{k+1}(z)\right) \cdots h\left(\varphi_{n_{0}}(z)\right) f\left(\varphi_{n_{0}+1}(z)\right),
\end{array}
$$

and $f\left(\varphi_{k}(z)\right)=0$ since $\lambda \notin\{0\} \cup \mathcal{S}$. Once again, by (44), we derive that

$$
\lambda^{k} f(z)=h(z) h(\varphi(z)) \cdots h\left(\varphi_{k-1}(z)\right) f\left(\varphi_{k}(z)\right)=0,
$$

and $f(z)=0$. 
Case III ( $\mathscr{F} \subset X \backslash X_{0}, \mathscr{F}$ is infinite and $\mathscr{N}$ is infinite). Notice that $\left\{\varphi_{n}(z): n \in \mathcal{N}\right\} \subset\left(X \backslash X_{0}\right) \cap \varphi\left(X \backslash X_{0}\right)$. Since $\varphi(X \backslash$ $\left.X_{0}\right)$ is totally bounded, we can derive that $\left\{\varphi_{n}(z): n \in \mathcal{N}\right\}$ converges to a point $\bar{x} \in X$. Moreover, $\varphi_{n}(z) \rightarrow \bar{x}$ since $\varphi$ is supercontractive. Then we have that $|h(\bar{x})| \geq \delta_{0}$ and $\varphi(\bar{x})=\bar{x}$. By (44) we can see that $f(\bar{x})=0$. Since $\varphi$ is supercontractive, there exists $\delta_{1}>0$ such that

$$
\begin{array}{r}
d\left(\varphi\left(z_{1}\right), \varphi\left(z_{2}\right)\right)<\frac{|\lambda|}{2\|h\|_{\infty}} d\left(z_{1}, z_{2}\right) \\
\text { when } 0<d\left(z_{1}, z_{2}\right)<\delta_{1} .
\end{array}
$$

Choose $N \in \mathbb{N}$ such that $d\left(\varphi_{n}(z), \bar{x}\right)<\delta_{1}$ for all $n \geq N$, and we have, for any $n \in \mathbb{N}$, that

$$
\begin{aligned}
\left|\lambda^{n} f\left(\varphi_{N}(z)\right)\right|= & \mid h\left(\varphi_{N}(z)\right) h\left(\varphi_{N+1}(z)\right) \cdots \\
& h\left(\varphi_{N+n-1}(z)\right) f\left(\varphi_{n+N}(z)\right) \mid \\
\leq & \|h\|_{\infty}^{n}\left|f\left(\varphi_{n+N}(z)\right)\right| \\
= & \|h\|_{\infty}^{n}\left|f\left(\varphi_{n+N}(z)\right)-f(\bar{x})\right| \\
\leq & \|h\|_{\infty}^{n} L(f) d\left(\varphi_{n+N}(z), \bar{x}\right) \\
\leq & \|h\|_{\infty}^{n} L(f) \frac{|\lambda|}{2\|h\|_{\infty}} d\left(\varphi_{n+N-1}(z), \bar{x}\right) \\
\leq & \cdots \leq\|h\|_{\infty}^{n} L(f)\left(\frac{|\lambda|}{2\|h\|_{\infty}}\right)^{n} d\left(\varphi_{N}(z), \bar{x}\right) \\
= & L(f)\left(\frac{|\lambda|}{2}\right)^{n} d\left(\varphi_{N}(z), \bar{x}\right) .
\end{aligned}
$$

That is,

$$
\left|f\left(\varphi_{N}(z)\right)\right| \leq \frac{1}{2^{n}} L(f) d\left(\varphi_{N}(z), \bar{x}\right) .
$$

Since $n$ is arbitrary, we can derive that $f\left(\varphi_{N}(z)\right)=0$, and then $f(z)=0$ since $(44)$.

Case IV $\left(\mathscr{F} \subset X \backslash X_{0}, \mathscr{F}\right.$ is infinite and $\mathscr{N}$ is finite). We can choose $N_{0} \in \mathbb{N}$ such that $\left|h\left(\varphi_{n}(z)\right)\right|<\delta_{0}$ for $n>N_{0}$. From (44), we have that

$$
\begin{array}{rl}
\lambda^{n} & f(z) \\
& =h(z) h(\varphi(z)) \cdots h\left(\varphi_{n-1}(z)\right) f\left(\varphi_{n}(z)\right),
\end{array}
$$

and then

$$
|f(z)| \leq\|h\|_{\infty}^{N_{0}} \delta_{0}^{-N_{0}}\left(\frac{\delta_{0}}{\lambda}\right)^{n}\|f\|_{\infty},
$$

for all $n>N_{0}$. This implies that $f(z)=0$ as $\delta_{0}<|\lambda|$.

\section{Acknowledgments}

The authors would like to express their thanks to the referees for several helpful comments which improved the presentation of this paper. Research of the second author was partially supported by NSF of China $(11301285,11371201)$. The fourth author was supported by Department of Applied Mathematics and the Research Group for Nonlinear Analysis and Optimization, post-doctoral fellowship at the National Sun Yat-sen University when this work was started, and this research is supported in part by Taiwan NSC grant 102-2115M-033-006.

\section{References}

[1] M. I. Garrido and J. A. Jaramillo, "Variations on the BanachStone theorem," Extracta Mathematicae, vol. 17, no. 3, pp. 351$383,2002$.

[2] K. Jarosz and V. D. Pathak, "Isometries and small bound isomorphisms of function spaces," in Function Spaces, K. Jarosz, Ed., vol. 136 of Lecture Notes in Pure and Applied Mathematics, pp. 241-271, Marcel Dekker, New York, NY, USA, 1992.

[3] H. Kamowitz, "Compact weighted endomorphisms of $C(X)$," Proceedings of the American Mathematical Society, vol. 83, no. 3, pp. 517-521, 1981.

[4] J. Cao, I. Reilly, and H. Xiong, "A lattice-valued Banach-Stone theorem," Acta Mathematica Hungarica, vol. 98, no. 1-2, pp. 103110, 2003.

[5] J.-X. Chen, Z. L. Chen, and N. Wong, "A banach-stone theorem for riesz isomorphisms of banach lattices," Proceedings of the American Mathematical Society, vol. 136, no. 11, pp. 3869-3874, 2008.

[6] Z. Ercan and S. Önal, "Banach-stone theorem for Banach lattice valued continuous functions," Proceedings of the American Mathematical Society, vol. 135, no. 9, pp. 2827-2829, 2007.

[7] Z. Ercan and S. Önal, "The Banach-Stone theorem revisited," Topology and its Applications, vol. 155, no. 16, pp. 1800-1803, 2008.

[8] X. Miao, J. Cao, and H. Xiong, "Banach-Stone theorems and Riesz algebras," Journal of Mathematical Analysis and Applications, vol. 313, no. 1, pp. 177-183, 2006.

[9] M. I. Garrido and J. A. Jaramillo, "Homomorphisms on function lattices," Monatshefte fur Mathematik, vol. 141, no. 2, pp. 127-146, 2004.

[10] M. I. Garrido and J. A. Jaramillo, "Lipschitz-type functions on metric spaces," Journal of Mathematical Analysis and Applications, vol. 340, no. 1, pp. 282-290, 2008.

[11] N. Weaver, "Lattices of Lipschitz functions," Pacific Journal of Mathematics, vol. 164, pp. 179-193, 1994.

[12] A. Jiménez-Vargas and M. Villegas-Vallecillos, "Order isomorphisms of little Lipschitz algebras," Houston Journal of Mathematics, vol. 34, no. 4, pp. 1185-1195, 2008.

[13] A. Jiménez-Vargas, A. M. Campoy, and M. Villegas-Vallecillos, "The uniform separation property and banach-stone theorems for lattice-valued lipschitz functions," Proceedings of the American Mathematical Society, vol. 137, no. 11, pp. 3769-3777, 2009.

[14] L. Dubarbie, "Maps preserving common zeros between subspaces of vector-valued continuous functions," Positivity, vol.14, no. 4, pp. 695-703, 2010.

[15] J. Araujo and L. Dubarbie, "Biseparating maps between Lipschitz function spaces," Journal of Mathematical Analysis and Applications, vol. 357, no. 1, pp. 191-200, 2009.

[16] L. Li and D. Leung, "Order isomorphisms on function space," Studia Mathematica. In press. 
[17] L. Li and N.-C. Wong, "Kaplansky theorem of completely regular spaces," Proceedings of the American Mathematical Society. In press.

[18] N. Weaver, Lipschitz Algebras, World Scientific Pbulishing, Singapore, 1999.

[19] H. Kamowitz and S. Scheinberg, "Some properties of endomorphisms of Lipschitz algebras," Studia Mathematica, vol. 96, no. 3, pp. 383-391, 1990.

[20] A. Jiménez-Vargas and M. Villegas-Vallecillos, "Compact composition operators on noncompact Lipschitz spaces," Journal of Mathematical Analysis and Applications, vol. 398, no. 1, pp. 221229, 2013. 


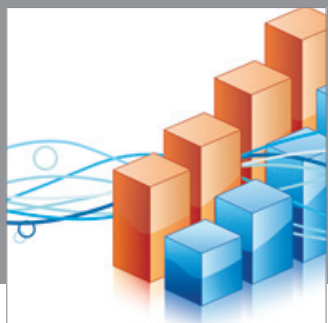

Advances in

Operations Research

mansans

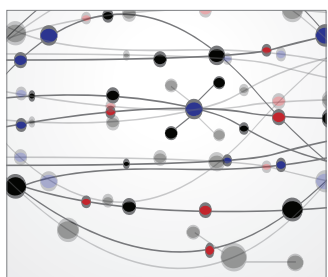

The Scientific World Journal
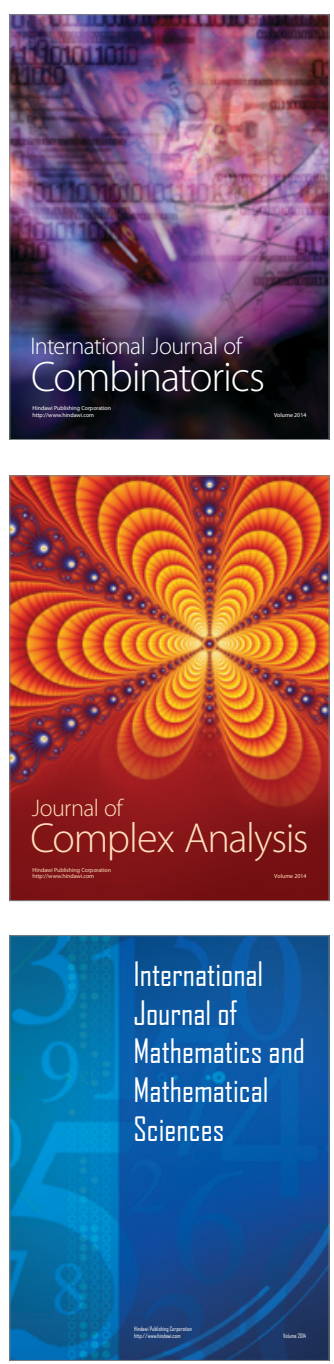
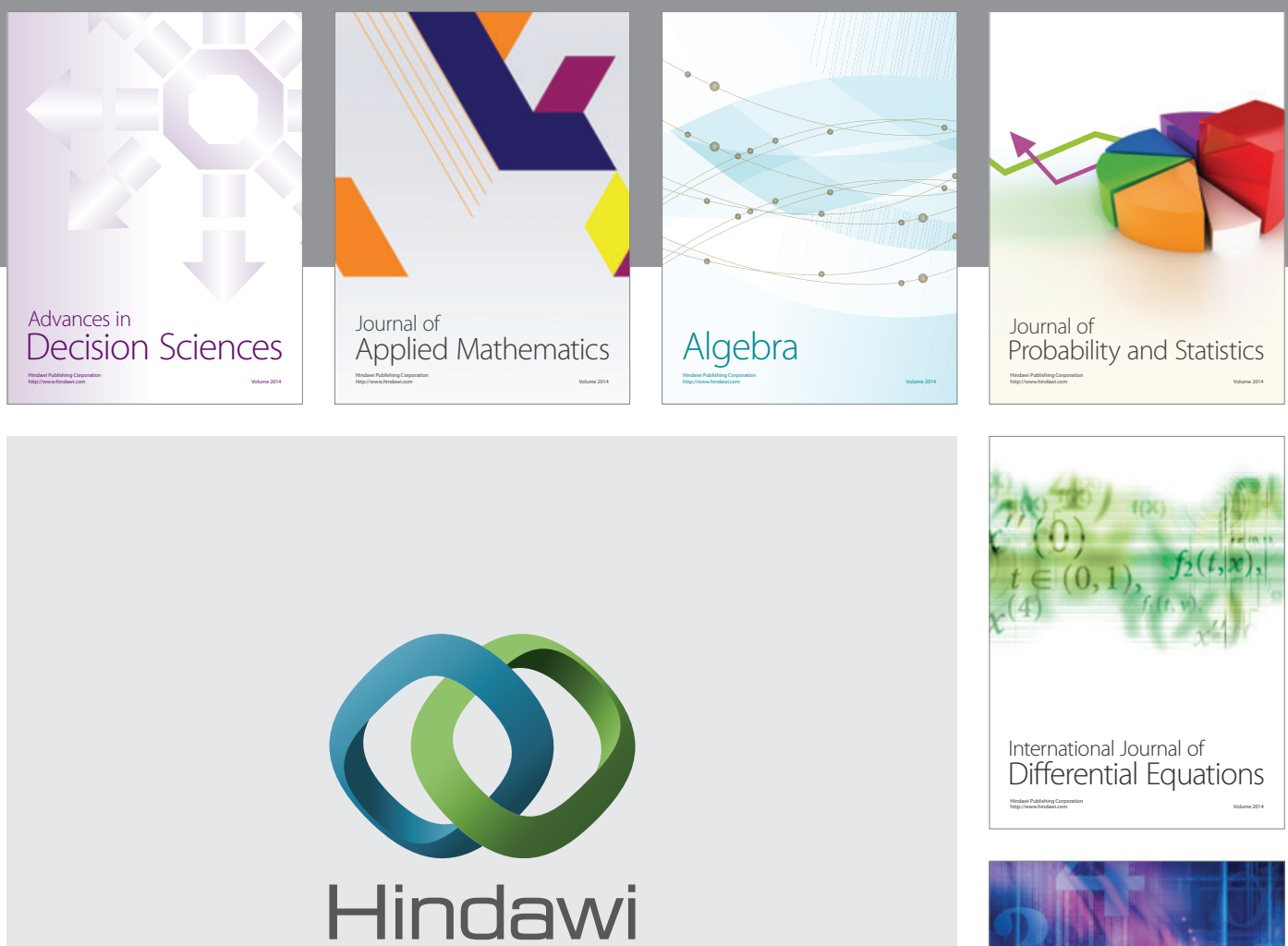

Submit your manuscripts at http://www.hindawi.com
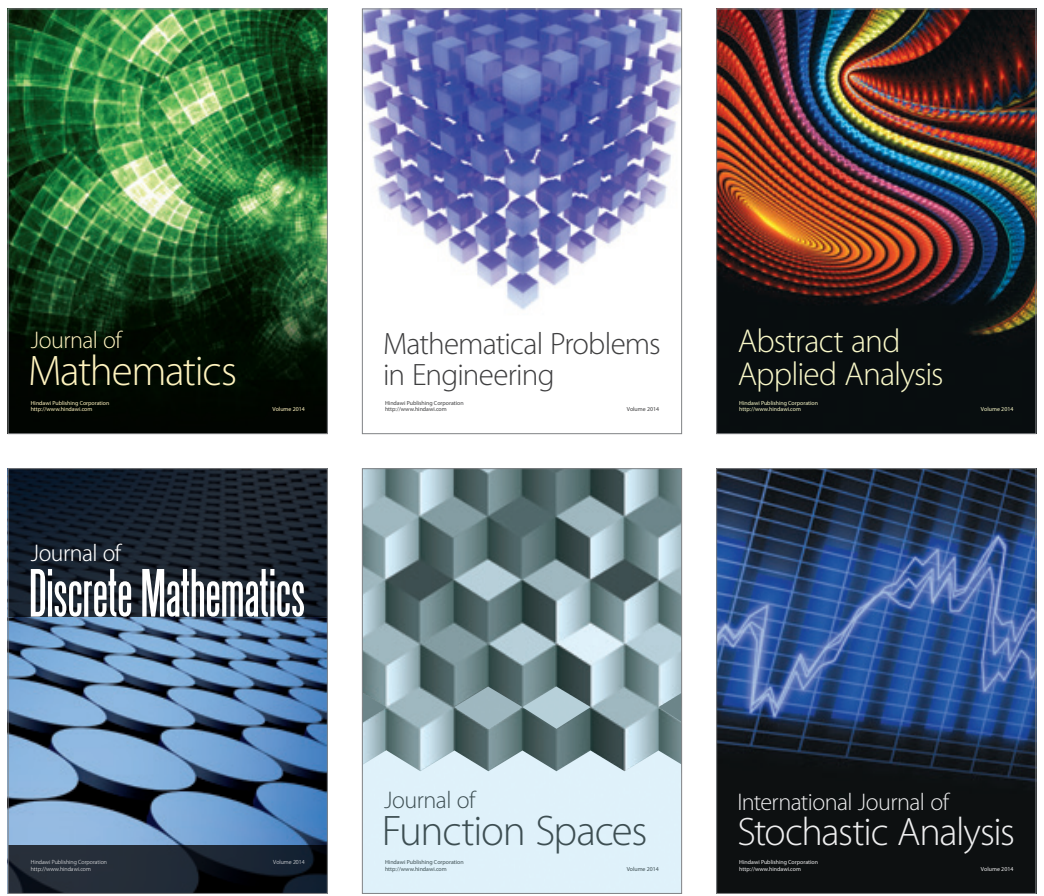

Journal of

Function Spaces

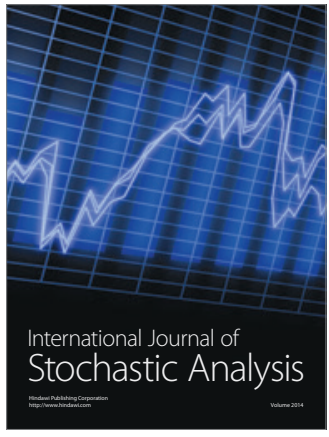

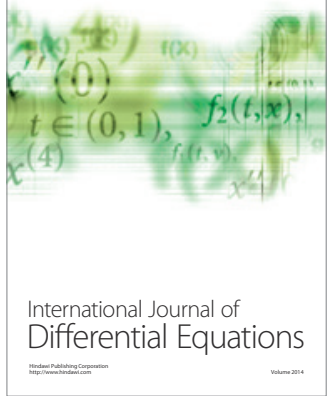
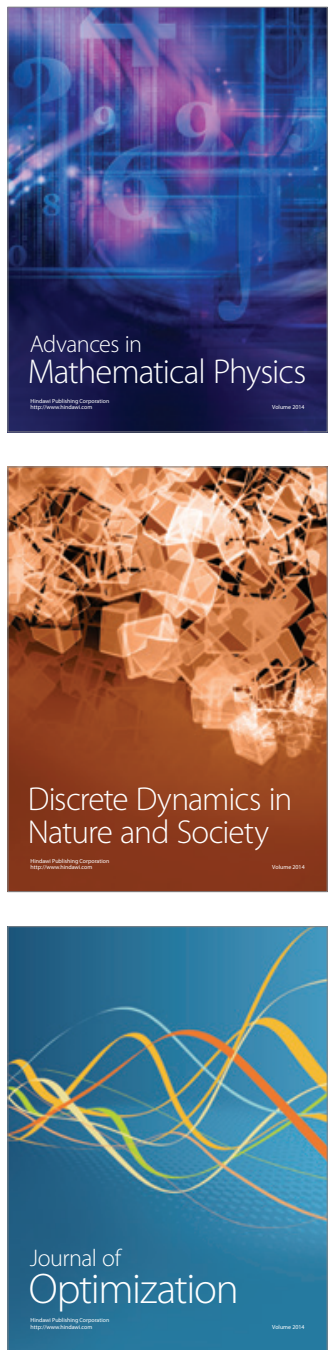